\title{
An Integral Transformation and its Applications to Harmonic Analysis on the Space of Solutions of Heat Equation
}

\author{
By \\ Soon-Yeong Chung* and Yongjin Yeom**
}

\begin{abstract}
We introduce an integral transformation $T$ defined by

$$
(T f)(x, t)=\int e^{-i x y-t|y| 2} f(y) d y, \quad f \in L^{1}\left(R^{n}\right)
$$

in order to do harmonic analysis on the space of $C^{\infty}$ solutions of heat equation.

First, the Paley-Wiener type theorem for the transformation $T$ will be given for the $C^{\infty}$ functions and distributions with compact support.

Secondly, as an application of the transformation the solutions of heat equation given on the torus $\boldsymbol{T}^{n}$ will be characterized.

Finally, we represent solutions of heat equation as an infinite series of Hermite temperatures, which are to be defined as the images of Hermite polynomials under the transformation $T$.
\end{abstract}

\section{§1. Introduction}

By a temperature we mean a $C^{\infty}$ solutions of the heat equation. In this paper we will do harmonic analysis on the space of solutions of heat equation $\left(\partial_{t}-\Delta\right) u(x, t)=0$ on $\boldsymbol{R}^{n} \times(0, T)$.

It is well known that the Fourier transform plays an important role in the usual harmonic analysis. In order to do harmonic analysis on the temperatures we need some integral transformation which maps the usual functions or generalized functions on $\boldsymbol{R}^{n}$ to temperatures. A point of this paper is to combine advantages of the Fourier transform and standard properties of temperatures so that we can obtain several parallel results as we might see

Communicated by T. Kawai, December 28, 1998. 'Revised April 16, 1999.

1991 Mathematics Subject Classifications: Primary 35K05 ; Secondary 42B10, 46F05.

* Department of Mathematics, Sogang University, Seoul 121-742, Korea. e-mail: sychung @ccs.sogang.ac.kr

** Department of Mathematics, Seoul National University, Seoul 151-742, Korea. e-mail: yjyeom @ math.snu.ac.kr 
in the usual harmonic analysis.

In this paper we first introduce an integral transformation $T$ as a variant of the Fourier transform, which is defined by

$$
(T f)(x, t)=\int e^{-i x y-t|y| 2} f(y) d y, f \in L^{1} .
$$

We introduce several interesting properties of such an integral transformation $T$ which will be widely used throughout this paper.

As the first main theorem we shall give a Palay-Wiener type theorem of this integral transformation $T$. Especially, $C^{\infty}$ functions with compact support and distributions with compact support will be characterized by entire temperatures (see Definition 3.1) respectively, via their images of this integral transformation $T$.

Next, we shall characterize periodic temperatures on $\boldsymbol{R}^{n} \times(0, T)$ or temperatures on the $n$-dimensional torus $T^{n}$ without any condition on their initial temperatures. The initial temperature of any temperature on the torus will be identified with the help of the integral transformation $T$ in an easy way.

In the last section, we introduce the Hermite temperature $\mathscr{H}_{n}(x, t)$ as an image of the Hermite polynomial under the integral transformation $T$. Then it will be shown that Hermite temperature has a similar orthogonal property and can be used to expand the temperatures with some growth condition into infinite series of Hermite temperatures.

\section{§2. An Integral Transformation $T$}

We start with a fundamental space on which we introduce the integral transformation. For $1 \leq p \leq \infty$ the space $L^{p}(d \mu)$ is the set of all functions whose $p$-th power is Lebesgue integrable with respect to the measure $\mu$ on $\boldsymbol{R}^{n}$. By $\mathscr{L}^{p}\left(\boldsymbol{R}^{n}\right)$ or simply $\mathscr{L}^{p}$ we denote the set of all functions $f$ such that for every $\epsilon>0, f$ belongs $L^{p}\left(e^{-\varepsilon|x|^{2}} d x\right)$, where $d x$ is the Lebesgue measure. Then it is easy to see that

$$
L^{p}(d x) \subset \mathscr{L}^{p}
$$

for each $p \geq 1$. The topology is given on the space $\mathscr{L}^{p}\left(\boldsymbol{R}^{n}\right)$ by the convergence that $f_{j} \rightarrow 0$ in $\mathscr{L}^{p}\left(\boldsymbol{R}^{n}\right)$ if and only if for every $\epsilon>0, f_{j} \rightarrow 0$ in $L^{p}\left(e^{-\varepsilon|x|^{2}} d x\right)$. In fact, the topology is the projective limit of $L^{p}\left(e^{-\varepsilon|x|^{2}} d x\right)$ as $\varepsilon \rightarrow 0$. i.e.

$$
\mathscr{L}^{p}\left(\boldsymbol{R}^{n}\right)=\lim _{\varepsilon \rightarrow 0} L^{p}\left(e^{-\varepsilon|x|^{2}} d x\right)
$$


Now we introduce the integral transformation as follows:

Definition 2.1. For $f \in \mathscr{L}^{1}\left(\boldsymbol{R}^{n}\right)$ and $t>0$ we difine

$$
(T f)(x, t)=\int e^{-i x y-t|y|^{2}} f(y) d y
$$

From this we can easily see that $(T f)(x, t)$ satisfies the heat equation

$$
\left(\partial_{t}-\Delta\right)(T f)(x, t)=0 \text { on } R^{n} \times(0, \infty) .
$$

Definition 2.2. By $\mathscr{T}\left(\boldsymbol{R}_{+}^{n+1}\right)$ we denote the set of all infinitely differentiable solutions $u(x, t)$ of the heat equation $\left(\partial_{t}-\Delta\right) u(x, t)=0$ on $\boldsymbol{R}^{n} \times(0, \infty)$ and we call its element a temperature on $\boldsymbol{R}_{+}^{n+1}$. We give a topology on $\mathscr{T}\left(\boldsymbol{R}_{+}^{n+1}\right)$ with the projective limit of the topology given by the semi-norms

$$
\|u(x, t)\|_{\infty, t}=\sup _{x \in \mathbb{R}}|u(x, t)|
$$

for each $t>0$. Then we have the following:

Theorem 2.3. The integral transformation $T: \mathscr{L}^{1}\left(\boldsymbol{R}^{n}\right) \rightarrow \mathscr{T}\left(\boldsymbol{R}_{+}^{n+1}\right)$ given by (2.1) is linear, injective, and continuous. Moreover, we have

$$
\sup _{x \in R^{n}}|(T f)(x, t)| \leq\|f\|_{L^{1}\left(e^{-t|x| 2} d x\right)}
$$

for each $t>0$.

The transformation $T$ plays a similar role as the Fourier transform in the usual harmonic analysis, since it is actually the Fourier transform of $f$ after multiplication by the Gaussian function $e^{-t|x|^{2}}$. Thus the transformation $T$ can be extended to the generalized function in a natural way. For example, for a tempered distribution $u \in \mathscr{S}^{\prime}$ the temperature $(T u)(x, t)$ is defined by

$$
(T u)(x, t)=\left\langle u, e^{-i x y-t|y|^{2}}\right\rangle, \quad t>0
$$

by considering the function $e^{-i x y-t|y|^{2}}$ as a test function.

Now we present some properties of the transformation $T$, which will be used later. From now on $*$ denotes the convolution product with respect to $x$ variable.

(i) For $f \in L^{1}\left(\boldsymbol{R}^{n}\right)$ 


$$
\begin{aligned}
(T f)(x, t) & =\left[e^{-t|y|^{2}} f(y)\right]^{\wedge}(x) \\
& =\int E(x-y, t) \hat{f}(y) d y
\end{aligned}
$$

where ${ }^{\wedge}$ denotes the Fourier transform and $E(x, t)$ denotes a fundamental solution of the heat operator $\partial_{t}-\Delta$.

(ii)

$$
\begin{aligned}
\left(T x^{\alpha}\right)(x, t) & =E(x, t) *(-1)^{|\alpha|} D^{\alpha} \delta \\
& =(-1)^{|\alpha|} D^{\alpha} E(x, t),
\end{aligned}
$$

where $\delta$ is the Dirac measure and $D^{\alpha}=\frac{1}{i^{|\alpha|}} \partial^{\alpha}$. More generally, if $P(x)=\sum_{|\alpha| \leq m} a_{\alpha} x^{\alpha}$ is a polynomial, then

$$
[T P(x)](x, t)=P(-D) E(x, t) .
$$

(iii) For a function $f$ and a distribution $u$ the translation $\tau_{h}$ by $h \in \mathbb{R}^{n}$ is defined by

$$
\left(\tau_{h} f\right)(x)=f(x-h)
$$

and

$$
\begin{aligned}
\left\langle\tau_{h} u, \phi\right\rangle & =\left\langle u, \tau_{-h} \phi\right\rangle \\
& =\langle u, \phi(x+h)\rangle
\end{aligned}
$$

for any test functions $\phi(x)$ corresponding to $u$. Then for $u \in \mathscr{S}^{\prime}$ we have

$$
\left[T\left(\tau_{h} f\right)\right](x, t)=\left\langle\hat{u}_{\xi}, e^{-i h \xi} E(x-\xi, t)\right\rangle .
$$

(iv) For $u \in \mathscr{S}^{\prime}$ we have

$$
T\left(x^{\alpha} u\right)=(-1)^{\alpha} D^{\alpha}(T u),
$$

and

$$
T(P(x) u)=P(-D) T u,
$$

where $P(x)$ is a polynomial.

(v) For $u \in \mathscr{S}^{\prime}$ and $\phi \in \mathscr{S}$ we have

$$
\begin{aligned}
T(\phi u) & =(T u) * \hat{\phi} /(2 \pi)^{n} \\
& =(T \phi) * \hat{u} /(2 \pi)^{n} .
\end{aligned}
$$

But, we should note here that 


$$
T(\phi * u)=E(x, t) * \hat{\phi} \hat{u} .
$$

(vi) Using the semigroup property of the heat kernel $E(x, t)$ we obtain

$$
(T u)(x, t+s)=E(x, t) *(T u)(x, s)
$$

for $t>0$ and $s>0$ when $u \in \mathscr{S}^{\prime}$.

(vii) An analogue of Parseval's identity for the transformation $T$ can be derived as follows: For $f$ and $g$ in $\mathscr{L}^{2}\left(\boldsymbol{R}^{n}\right)$

$$
\begin{aligned}
& \int(T f)(x, t) \overline{(T g)(x, t)} d x=\frac{1}{(2 \pi)^{n}} \widehat{(T f)} \widehat{\widehat{(T g)}} d x \\
& =\frac{1}{(2 \pi)^{n}} \int \widehat{E * \hat{f}} \widehat{E^{*} \hat{g}} d x \\
& =(2 \pi)^{n} \int f(-x) \overline{g(-x)} e^{-2 t|x|^{2}} d x \\
& =(2 \pi)^{n} \int f(x) \overline{g(x)} e^{-2 t|x|^{2}} d x \\
& =\langle f, g\rangle_{L^{2}\left(e^{-t|x|^{2}} d x\right)} .
\end{aligned}
$$

(viii) For $f \in L^{1}\left(\boldsymbol{R}^{n}\right)$ we can take a (pointwise) limit

$$
\begin{aligned}
(T f)(x, 0+) & =\lim _{t \rightarrow 0+} \int e^{-i x y-t|y|^{2}} f(y) d y \\
& =\int e^{-i x y} f(y) d y .
\end{aligned}
$$

Thus $(T f)(x, t)$ is a temperature whose initial value is just $\hat{f}(x)$, so that the integral transformation can be regarded as a generalization of the Fourier transform.

\section{§3. Paley-Wiener Type Theorems}

The Paley-Wiener theorem characterizes distributions or functions by the growth of their Fourier-Laplace transforms. Then it will be quite interesting to consider an analogue for the integral transformation $T$ introduced in the previous section.

Let $K$ be a compact subset of $R^{n}$ and $u \in \mathscr{E}^{\prime}(K)$. Then since the function $e^{-i y \xi-t|y|^{2}}$ is (real) analytic everywhere in $\boldsymbol{R}^{n}$ the transformation $T$ 


$$
(T u)(\zeta, t)=\left\langle u_{y}, e^{-i y \zeta-t|y|^{2}}\right\rangle, \zeta \in C^{n}
$$

is well defined. Moreover, it is easy to see the followings:

(i) $(T u)(\zeta, t)$ is an entire function of $\zeta$ in $C_{\zeta}^{n}$.

(ii) $\left(\partial_{t}-\Delta_{\zeta}\right)(T u)(\zeta, t)=0$, on $C_{\zeta}^{n} \times(0, \infty)$.

Definition 3.1. An infinitely differentiable function $u(\zeta, t)$ on $\mathbb{C}^{n} \times(0, \infty)$ is called an entire temperature if it satisfies (i) and (ii) above.

Now we state the Paley-Wiener type theorem for the transformation $T$ of the distributions and functions with compact support.

Theorem 3.2. Let $K$ be a compact convex subset of $\mathbb{R}^{n}$.

(i) If $f \in C_{0}^{\infty}(K)$ then $(T f)(\zeta, t)$ is an entire temperature satisfying that for any $N>0$ there exists a constant $C>0$ such that

$$
|(T f)(\zeta, t)| \leq C\langle\zeta\rangle^{-N}\langle t\rangle^{N / 2} e^{H_{K}(\operatorname{Im} \zeta)}, \quad \zeta \in C^{n}, t>0,
$$

where $\langle x\rangle=\left\langle 1+|x|^{2}\right\rangle^{1 / 2}$ and $H_{K}(\xi)=\sup _{x \in \mathbf{K}}\langle x, \xi\rangle$.

Conversely, if $F(\zeta, t)$ is an entire temperature satisfying (3.1) then there exists a unique $f \in C_{0}^{\infty}(K)$ such that

$$
(T f)(\zeta, t)=F(\zeta, t) \text { on } C_{\zeta}^{n} \times(0, \infty) \text {. }
$$

(ii) If $u$ is a distribution of order $N$ with support in $K$ then $(T u)(\zeta, t)$ is an entire temperature satisfying that there exists a constant $C>0$ such that

$$
|(T u)(\zeta, t)| \leq C\langle\zeta\rangle^{N}\langle t\rangle^{N / 2} e^{H_{K}(\mathrm{Im} \zeta)}, \quad \zeta \in C^{n}, t>0 .
$$

Conversely, if $F(\zeta, t)$ is an entire temperature satisfying (3.2) then there exists a unique $u \in \mathscr{E}^{\prime}(K)$ such that $(T u)(\zeta, t)=F(\zeta, t)$ in $\mathbb{C}_{\zeta}^{\infty} \times(0, \infty)$.

Proof. The uniqueness is easily obtained applying the well known uniqueness theorems for temperatures (see [16]).

(i) First, we note that for a constant $C>0$

$$
\left|\partial_{x}^{\alpha} e^{-s|x|^{2}}\right| \leq C^{|\alpha|} S^{|\alpha| / 2} \alpha !^{1 / 2} \exp \left(-\frac{s}{2}|x|^{2}\right), \quad s>0, \quad x \in \boldsymbol{R}^{n} .
$$

This inequality can be easily obtained by use of Cauchy's integral formula. Then we have

$$
\zeta^{\alpha}(T f)(\zeta, t)=\int(-1)^{|\alpha|} D_{y}^{\alpha}\left(e^{-i \zeta y}\right) e^{-t|y|^{2}} f(y) d y
$$




$$
\begin{aligned}
& =\int e^{-i \zeta y} D_{y}^{\alpha}\left(e^{-t|y|^{2}} f(y)\right) d y \\
& =\int_{K} e^{-i \zeta y} \sum_{\beta \leq \alpha}\left(\begin{array}{l}
\alpha \\
\beta
\end{array}\right)\left(\partial_{y}^{\beta} e^{-t|y|^{2}}\right)\left(\partial^{\alpha-\beta} f(y)\right) d y .
\end{aligned}
$$

Thus, for any $N>0$ and $|\alpha| \leq N$ we obtain that

$$
\begin{aligned}
\left|\zeta^{\alpha}(T f)(\zeta, t)\right| & \leq e^{H_{K}(\operatorname{lm} \zeta)} \int_{K} \sum_{\beta \leq \alpha}\left(\begin{array}{l}
\alpha \\
\beta
\end{array}\right) C^{|\beta|} t^{|\beta| / 2} \beta !^{1 / 2} e^{-\frac{t}{2}|y|^{2}}\left|\partial^{\alpha-\beta} f(y)\right| d y \\
& \leq C_{1}^{|\alpha|}(1+t)^{|\alpha| / 2} \alpha !^{1 / 2} e^{H_{K}(\operatorname{lm} \zeta)} \int_{K}\left|\partial^{\alpha-\beta} f(y)\right| d y \\
& \leq C(N, f)\langle t\rangle^{N / 2} e^{H_{K}(\operatorname{lm} \zeta)}, \quad \zeta \in C^{n}
\end{aligned}
$$

for some constant $C_{1}>0$ and $C(N, f)>0$. Then it follows that

$$
|(T f)(\zeta, t)| \leq C\langle t\rangle^{N / 2}\langle\zeta\rangle^{-N} e^{H_{K}(\operatorname{lm} \zeta),} \quad \zeta \in C^{n}, t>0
$$

for a constant $C>0$.

Now we prove the converse. Define

$$
f(x)=e^{t|x|^{2}} \int e^{i x \xi} F(\xi, t) d \xi, \quad t>0 .
$$

The righthand side is independent of $t>0$, since

$$
\partial_{t}\left(e^{t|x|^{2}} \mathscr{F}_{\xi}^{-1}(F(\xi, t))\right)=0
$$

where $\mathscr{F}_{\xi}^{-1}$ is the inverse Fourier transform. This can be seen by taking the Fourier transform on $\left(\partial_{t}-\Delta_{\xi}\right) F(\xi, t)=0$. By the decay condition (3.1) we can see that $f(x)$ is a $C^{\infty}$ function and $(T f)(\zeta, t)=F(\zeta, t)$ for $\zeta \in C_{\zeta}^{n}$ and $t>0$. Now it remains to show that supp $f \subset K$. Fixing $t=t_{0}$ and shifting the path in (3.4) we have

$$
\begin{aligned}
|f(x)| & \leq e^{t_{0}|x|^{2}}\left|\int e^{i x(\xi+i \eta)} F\left(\xi+i \eta, t_{0}\right) d \xi\right| \\
& \leq C\left\langle t_{0}\right\rangle^{N / 2} e^{t_{0}|x|^{2}-x \eta+H_{K}(\eta)} .
\end{aligned}
$$


Now let $x \notin K$. Since $K$ is compact and convex the Hahn-Banach theorem gives a hyperplane $\left\{y \in \boldsymbol{R}^{n} \mid\left\langle y, \eta_{0}\right\rangle=c\right\}$ for some $\eta_{0} \in \boldsymbol{R}^{n}$ such that

$$
\left\langle y, \eta_{0}\right\rangle<c, \quad y \in K
$$

and

$$
\left\langle x, \eta_{0}\right\rangle>c .
$$

Then it follows that

$$
H_{K}\left(\eta_{0}\right)=\sup _{y \in K}\left\langle y, \eta_{0}\right\rangle\left\langle c<\left\langle x, \eta_{0}\right\rangle .\right.
$$

Taking $\eta=k \eta_{0}(k>0)$ in (3.5) we have

$$
|f(x)| \leq C\left\langle t_{0}\right\rangle^{N / 2} e^{-k_{0}|x|^{2}-k\left\{\left\langle x, \eta_{0}\right\rangle-H_{K}\left(\eta_{0}\right)\right\}} .
$$

Then the righthand side goes to 0 as $k$ goes to $\infty$, which implies that $f(x)=$ 0 . Thus we have supp $f \subset K$.

(ii) First, we choose a cut-off function $\chi_{\delta} \in C_{0}^{\infty}\left(K_{\delta}\right)$ for $0<\delta \leq 1$ where $K_{\delta}=\{x+y \mid x \in K$ and $|y| \leq \delta\}$, so that

$$
\chi_{\delta}(x)=1, \quad x \in K_{\delta / 2}
$$

and

$$
\left|\partial^{\alpha} \chi_{\delta}(x)\right| \leq C_{\alpha} \delta^{-|\alpha|}, \quad \alpha \in N_{0}^{n} .
$$

Since $\chi_{\delta} u=u$ and $u$ is a distribution of order $N$ we have

$$
\begin{aligned}
& |(T u)(\zeta, t)|=\left|\left\langle u, \chi_{\delta} e^{-i y \zeta-t|y|^{2}}\right\rangle\right| \\
& \leq C \sum_{|\alpha| \leq N} \sup _{y \in K_{\delta}}\left|\partial^{\alpha}\left(e^{-i y \zeta} \chi_{\delta} e^{t|y|^{2}}\right)\right| \\
& =C \sum_{|\alpha| \leq N} \sup _{y \in \mathbf{K}_{\delta} \beta \leq \alpha} \sum_{\beta}\left(\begin{array}{l}
\alpha \\
\beta
\end{array}\right)\left|\partial^{\alpha-\beta} e^{-i y \zeta}\right| \cdot\left|\partial^{\beta}\left(\chi_{\delta} e^{-t|y|^{2}}\right)\right| \\
& \leq C \sum_{|\alpha| \leq N}|\zeta|^{|\alpha-\beta|} \sup _{y \in K_{\delta}} e^{\langle y, \eta\rangle} \sum_{\gamma \leq \beta}\left(\begin{array}{l}
\beta \\
\gamma
\end{array}\right)\left|\partial^{\beta-\gamma} \chi_{\delta}(y)\right| \cdot\left|\partial^{\gamma} e^{-t|y|^{2}}\right|
\end{aligned}
$$

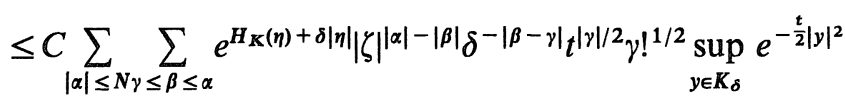

$$
\begin{aligned}
& \leq C\langle t\rangle^{N / 2} e^{H_{K}(\eta)+\delta|\eta|} \sum_{|\beta| \leq N} \delta^{-|\beta|}\langle\zeta\rangle^{N-|\beta|},
\end{aligned}
$$


where $C$ denotes a constant which is not essentially the same in each step.

Then if we take $\delta=1 / 1+|\zeta|$ we obtain

$$
|(T u)(\zeta, t)| \leq C\langle\zeta\rangle^{N}\langle t\rangle^{N / 2} e^{H_{K}(\eta)}
$$

which is required.

Now we prove the converse. Since $F(\cdot, t)$ is a tempered distribution for each $t>0$ we can take the inverse Fourier transform $\mathscr{F}_{\xi}^{-1}$ with respect to $\xi$ variable. Put

$$
u=e^{-t|x|^{2}} \mathscr{F}_{\xi}^{-1} F(\cdot, t)
$$

Then $u$ is also a tempered distribution and does not depend on the parameter $t>0$ by the same argument before. Moreover, it is true that $(T u)(\zeta, t)=F(\zeta, t)$ for all $\zeta \in C^{n}$ and $t>0$.

On the other hand, it follows from (3.2) that the usual Paley-Wiener theorem gives $\omega_{t} \in \mathscr{E}^{\prime}(K)$ for each $t>0$ such that

$$
\hat{\omega}_{t}(\zeta)=F(\zeta, t)=\left(e^{-t|x|^{2}} u\right)^{\wedge}(\zeta, t) .
$$

Therefore, we have $u=e^{t|x|^{2}} \omega_{t}$. But since $e^{t|x|^{2}} \neq 0$ everywhere and supp $\omega_{t} \subset K$ we can see that supp $u \subset K$ which completes the proof.

\section{§4. Periodic Temperatures on the Tours $T^{n}$}

For a multi-index $\alpha \in N_{0}^{n}$ by $|\alpha|$ we have denoted $|\alpha|=\alpha_{1}+\alpha_{2}+\cdots+\alpha_{n}$. But for $\alpha \in Z^{n},\|\alpha\|$ denotes the Euclidean length i.e. $\|\alpha\|^{2}=\alpha_{1}^{2}+\alpha_{2}^{2}+\cdots+\alpha_{n}^{2}$ throughout this section where $Z$ is the set of all integers.

As an application of the transformation $T$ we shall give a characterization of periodic temperatures on $\boldsymbol{R}^{\boldsymbol{n}} \times(0, T)$. Here by a periodic temperature we mean the temperature $u(x, t)$ such that

$$
u(x+\alpha, t)=u(x, t) \quad \text { on } \boldsymbol{R}^{n} \times(0, T)
$$

for any $\alpha \in Z^{n}$. A periodic temperature can be regarded as a temperature on the $n$-dimensional torus $T^{n}$. In fact, it is well known that if $u(x, t)$ is a periodic temperature on $\boldsymbol{R} \times(0, T)$ with the initial temperature $f(x)=u(x, 0)$ which is integrable on $(0,1)$, then $u(x, t)$ can be represented by the Fourier series 


$$
u(x, t)=\sum_{n=-\infty}^{\infty} a_{n} e^{-4 \pi^{2} n^{2} t+2 \pi i n x},
$$

where

$$
a_{n}=\int_{0}^{1} f(x) e^{-2 \pi i n x} d x
$$

Here, we shall characterize arbitrary periodic temperature on $\boldsymbol{R}^{n} \times(0, T)$, without any condition on its initial temperature. More precisely, the initial temperature of any periodic temperature will be given a meaning via the integral transformation $T$.

In oreder to do this we first introduce some space of functions of certain decay rate.

Definition 4.1. By $\mathscr{T}_{\frac{1}{2}}$ we denote the set of all $C^{\infty}$ functions satisfying the following: for every $\alpha \in N^{n}$ there exists $a>0$ such that

$$
\|\phi\|_{\alpha, a}=\sup _{x \in \mathbb{R}^{n}}\left|\partial^{\alpha} \phi(x)\right| e^{a|x|^{2}}
$$

is finite and by $\mathscr{T}_{\frac{1}{2}}^{\prime}$ we denote the strong dual space of $\mathscr{T}_{\frac{1}{2}}$ with respect to the topology defined by the norm (4.3).

Remark. (i) Each element $u$ of the space $\mathscr{T}_{\frac{1}{2}}^{\prime}$ belongs to the space of Schwartz distributions, which is defined by the set of all continuous linear functionals on the space $C_{0}^{\infty}$. In fact, it can be represented by $u=\partial^{\alpha} f(x)$ for some $\alpha$ and a continuous function $f(x)$ on $\boldsymbol{R}^{n}$ such that for some $a>0$ and $C>0$

$$
|f(x)| \leq C e^{a|x|^{2}}, \quad x \in \boldsymbol{R}^{n} .
$$

This can be proved by the same method which was used for the structure theorem for tempered distributions (see [13]).

(ii) It is easy to see that $(T \phi)(\cdot, t) \in \mathscr{T}_{\frac{1}{2}}$ for each $t>0$ and $\phi \in \mathscr{T}_{\frac{1}{2}}$. Therefore, the transformation $T$ on $\mathscr{T}_{\frac{1}{2}}^{\prime}$ is also well defined as, for $u \in \mathscr{T}_{\frac{1}{2}}^{\prime}$,

$$
(T u, \phi)=(u,(T \phi)(\cdot, t)), \quad \phi \in \mathscr{T}_{\frac{1}{2}} .
$$

(iii) The space $\mathscr{T}_{\frac{1}{2}}$ is a little different from the space $S_{\frac{1}{2}}$ which was introduced by Gelfand-Shilov([6]). But using the same arguments in [6] we can see that the Fourier transform is an isomorphism between $\mathscr{T}_{\frac{1}{2}}$ and the 
space $\mathscr{T}^{\frac{1}{2}}$ given by the set of all $C^{\infty}$ functions $\phi$ satisfying that for any $\beta \in N_{0}^{\beta}$ there exist $h>0$ and $C>0$ such that

$$
\sup _{x \in R^{n}}\left|x^{\beta} \partial^{\alpha} \phi(x)\right| \leq C h^{|\alpha|} \alpha !^{\frac{1}{2}}, \quad \alpha \in N_{0}^{n}
$$

i.e. $\hat{\mathscr{T}}=\mathscr{T}^{\frac{1}{2}}$

Now we are in a position to state the main theorem in this section.

Theorem 4.2. Let $u(x, t)$ be a periodic temperature on $\boldsymbol{R}^{n} \times(0, T)$. Then we can find a unique distribution $v(x)=\Sigma_{\alpha \in \mathbf{Z}^{n}} c_{\alpha} \delta(x+2 \pi \alpha) \in \mathscr{T}_{\frac{1}{2}}^{\prime}$ such that

$$
(T v)(x, t)=u(x, t)
$$

where $c_{\alpha}$ 's are complex numbers satisfying the following: for every $\epsilon>0$

$$
\left|c_{\alpha}\right| \leq C_{\varepsilon} e^{\varepsilon\|\alpha\|^{2}}, \quad \alpha \in Z^{n}
$$

Moreover, $u(x, t)$ can be uniquely expressed as

$$
u(x, t)=\sum_{\alpha \in \mathbf{Z}^{n}} c_{\alpha} e^{-4 \pi^{2}\|\alpha\|^{2} t+2 \pi i \alpha x}, \quad(x, t) \in \boldsymbol{R}^{n} \times(0, T)
$$

where

$$
c_{\alpha}=e^{4 \pi^{2}\|\alpha\|^{2} t} \int_{0}^{1} \cdots \int_{0}^{1} u(x, t) e^{-2 \pi i \alpha x} d x, \quad \alpha \in Z^{n}
$$

Proof. Since $u(\cdot, t)$ is periodic for each $t \in(0, T)$ it can be written as its Fourier series expansion

$$
u(x, t)=\sum_{\alpha \in \mathbf{Z}^{n}} b(\alpha, t) e^{2 \pi i \alpha x}, \quad x \in \boldsymbol{R}^{n}
$$

for each $t \in(0, T)$, where

$$
b(\alpha, t)=\int_{0}^{1} \ldots \int_{0}^{1} u(x, t) e^{-2 \pi i \alpha x} d x \quad \alpha \in Z^{n} .
$$

Moreover, since $u(\cdot, t)$ is $C^{\infty}$ in $\boldsymbol{R}^{n}$ it follows that for any $N>0$

$$
|b(\alpha, t)| \leq b_{N}(t)(1+|\alpha|)^{-N}, \quad \alpha \in Z^{n}
$$

for some constant $b_{N}(t)$ depending on $N$ and $t$.

We now apply the heat operator $\partial_{t}-\Delta$ to (4.7). In view of the condition 
(4.9) the Laplace operator $\Delta$ and the seminorm in (4.7) can be interchanged in their order of applications. But it may be nontrivial whether $\partial_{t}$ and the summation should be also interchanged. But since $\partial_{t} u(x, t)$ is also a periodic temperature if we use the decay condition and the uniqueness of the coefficients, they can be also interchangeable. Then,

$$
\begin{aligned}
\left(\partial_{t}-\Delta\right) u(x, t) & =\sum_{\alpha \in \mathbb{Z}_{0}^{n}} \frac{\partial b(\alpha, t)}{\partial t} e^{2 \pi i \alpha x}+\sum_{\alpha \in \mathbb{Z}_{0}^{n}} 4 \pi^{2}\|\alpha\|^{2} b(\alpha, t) e^{2 \pi i \alpha x} \\
& =\sum_{\alpha \in \mathbb{Z}_{0}^{n}}\left\{\frac{\partial b(\alpha, t)}{\partial t}+4 \pi^{2}\|\alpha\|^{2} b(\alpha, t)\right\} e^{2 \pi i \alpha x} \\
& =0
\end{aligned}
$$

on $\boldsymbol{R}^{n} \times(0, T)$. Thus from the uniqueness of the Fourier coefficients we obtain an ordinary differential equation

$$
\frac{d}{d t} b(\alpha, t)+4 \pi^{2}\|\alpha\|^{2} b(\alpha, t)=0, \quad t \in(0, T)
$$

for each $\alpha \in \mathbb{Z}^{n}$. Thus we have

$$
\frac{d}{d t}\left\{e^{4 \pi^{2}\|\alpha\|^{2} t} b(\alpha, t)\right\}=0
$$

which implies that the function $e^{4 \pi^{2}\|\alpha\|^{2} t} b(\alpha, t)$ is independent of $t>0$, so that we may write

$$
c_{\alpha}=e^{4 \pi^{2}\|\alpha\|^{2} t} b(\alpha, t) .
$$

This gives the expression (4.6). Moreover, it follows from (4.9) that for every $N>0$

$$
\left|c_{\alpha}\right| \leq b_{N}(t)(1+|\alpha|)^{-N} e^{4 \pi^{2}\|\alpha\|^{2 t}}, \quad 0<t<T .
$$

Since $c_{\alpha}$ is independent of $t>0$, we may choose $t$ arbitrarily, so that for every $\epsilon>0$ and some constant $C_{\varepsilon}>0$ we have

$$
\left|c_{\alpha}\right| \leq C_{\varepsilon} e^{\varepsilon\|\alpha\|^{2}}, \quad \alpha \in \mathbb{Z} .
$$

Now take $v(x)=\Sigma_{\alpha \in Z^{n}} c_{\alpha} \delta(x+2 \pi \alpha)$, where $c_{\alpha}$ 's are the constants given above. For any $\phi$ in $\mathscr{T}_{\frac{1}{2}}$ we have

$$
|\langle v, \phi\rangle|=\left|\sum_{\alpha \in \mathbb{Z}^{n}} c_{\alpha} \phi(-2 \pi \alpha)\right|
$$




$$
\begin{aligned}
& \leq \sum_{\alpha \in \mathbb{Z}^{n}} \mid c_{\alpha}\|\phi\|_{0, a} e^{-4 \pi^{2} a\|\alpha\|^{2}} \\
& \leq C_{\varepsilon}\|\phi\|_{0, a} \sum_{\alpha \in \mathbb{Z}^{n}} e^{\left(\varepsilon-4 \pi^{2} a\right)\|\alpha\|^{2}} \\
& \leq C_{1}\|\phi\|_{0, a},
\end{aligned}
$$

for some constant $C_{1}>0$ and by taking $\varepsilon>0$ so that $\varepsilon<4 \pi^{2} a$. Thus $v$ belongs to $\mathscr{T}_{\frac{1}{2}}^{\prime}$. Since

$$
\begin{aligned}
T[\delta(y+2 \pi \alpha)]= & \left\langle\delta(y+2 \pi \alpha), e^{-i y x-t|y|^{2}}\right\rangle \\
& =e^{-4 \pi^{2}\|\alpha\|^{2} t+2 \pi i \alpha x},
\end{aligned}
$$

we get for $(x, t) \in \boldsymbol{R}^{n} \times(0, T)$

$$
\begin{aligned}
(T v)(x, t) & =\sum_{\alpha \in \mathbb{Z}^{n}} c_{\alpha} e^{-4 \pi^{2}\|\alpha\|^{2} t+2 \pi i \alpha x} \\
& =u(x, t)
\end{aligned}
$$

On the other hand, the uniqueness in the (4.4) and (4.6) is easy.

Using the above result we can find the inital temperatures of arbitrary periodic temperatures. To be precise, the initial temperature of any periodic temperature belongs to the space $\left(\mathscr{T}^{\frac{1}{2}}\right)^{\prime}$. Namely, we have the following:

Theorem 4.3. Let $u(x, t)$ be a periodic temperature on $\boldsymbol{R}^{n} \times(0, T)$. Then there exists a unique $u_{0} \in\left(\mathscr{T}^{\frac{1}{2}}\right)^{\prime}$ such that

$$
u(x, t)=\left\langle u_{0} E(x-y, t)\right\rangle, \quad(x, t) \in \boldsymbol{R}^{n} \times(0, T) .
$$

Proof. By Theorem $4.2 u(x, t)$ can be written as $(T v)(x, t)=u(x, t)$ for a unique $v \in \mathscr{T}_{\frac{1}{2}}^{\prime}$. Thus it follows that

$$
\begin{aligned}
u(x, t) & =\left\langle v, e^{-i x y-t|y|^{2}}\right\rangle \\
& =\langle\hat{v}, E(x-y, t)\rangle .
\end{aligned}
$$

Taking $u_{0}=\hat{v}$ we can easily see that $u_{0}$ belongs to $\left(\mathscr{T}^{\frac{1}{2}}\right)^{\prime}$ (see Remark above) and $u(x, t)$ converges to $u_{0}=\hat{v}$ in $\left(\mathscr{T}^{\frac{1}{2}}\right)^{\prime}$ as $t \rightarrow 0$.

For a temperature $u(x, t)$ on $\boldsymbol{R}^{n} \times(0, T)$ we say that it has the Huygens 
property if

$$
u(x, t)=\int_{\mathbf{R}^{n}} E(x-y, t-s) u(y, s) d y
$$

for every $t$ and $s$ with $0<s<t<T$.

Corollary 4.4. Every periodic temperature on $\boldsymbol{R}^{n} \times(0, T)$ has the Huygens property there.

Proof. Using the semigroup property of $E$ i.e. )

$$
E\left(x, t_{1}+t_{2}\right)=E\left(x, t_{1}\right) * E\left(x, t_{2}\right), \quad t_{1}>0, t_{2}>0,
$$

it follows from (4.10) that

$$
\begin{aligned}
u(x, t) & =u_{0} * E(x, t) \\
& =u_{0} * E(x, s) * E(x, t-s) \\
& =u(x, s) * E(x, t-s) \\
& =\int_{\mathbf{R}^{n}} u(y, s) E(x-y, t-s) d y .
\end{aligned}
$$

In fact, the last integral converges since

$$
|u(x, t)| \leq C(N, t)|x|^{N}, \quad x \in R^{n}
$$

for some $N>0$ and $C(N, t)$ depending on $N>0$ and $t>0$

As an another application the backward Cauchy problem for the heat equation on the $n$-dimensional torus $T^{n}$ can be solved as follows:

Corollary 4.5. A solution of the backward Cauchy problem

$$
\left\{\begin{array}{l}
\left(\partial_{t}-\Delta\right) u(x, t)=0 \text { on } T^{n} \times\left(0, t_{0}\right) \\
u\left(x, t_{0}\right)=f_{0}(x), \quad x \in T^{n}
\end{array}\right.
$$

can be uniquely expressed as

$$
u(x, t)=\sum_{\alpha \in \mathbb{Z}^{n}} a_{\alpha} e^{-4 \pi^{2}\|\alpha\|^{2} t+2 \pi i \alpha x}, \quad x \in T^{n}, t>0,
$$

where

$$
a_{\alpha}=e^{4 \pi^{2}\|\alpha\|^{2} t_{0}} \int_{0}^{1} \ldots \int_{0}^{1} f_{0}(x) e^{-2 \pi i \alpha x} d x, \quad \alpha \in Z^{n}
$$




\section{$\S 5$. Hermite Temperatures}

The Hermite polynomial $H_{n}(x)$ of degree $n$ is defined by the Rodriguez formula

$$
H_{n}(x)=(-1)^{n} e^{x^{2}}\left(\frac{d}{d x}\right)^{n} e^{-x^{2}}, \quad x \in \boldsymbol{R}
$$

for $n=0,1,2, \ldots$ The orthogonality is defined by

$$
\begin{aligned}
\left\langle\left\langle H_{n}(x), H_{m}(x)\right\rangle\right\rangle: & =\int_{-\infty}^{\infty} H_{n}(x) H_{m}(x) e^{-x^{2}} d x \\
& = \begin{cases}2^{n} n ! \sqrt{\pi}, & \text { if } n=m \\
0, & \text { if } n \neq m .\end{cases}
\end{aligned}
$$

Then it is well known that if $e^{-\frac{x^{2}}{2}} f(x)$ is a tempered distribution, then we can write the distribution $f$ as

$$
f=\sum_{n=0}^{\infty} a_{n} H_{n}(x)
$$

where

$$
a_{n}=\frac{1}{2^{n} n ! \sqrt{\pi}}\left\langle f, e^{-x^{2}} H_{n}(x)\right\rangle .
$$

Moreover, the coefficients $a_{n}$ have an estimate that

$$
\left|a_{n}\right| \leq C(1+n)^{N}, \quad n \in N_{0}
$$

for some $N>0$.

Definition 5.1. For each $n \in N_{0}$ we call the temperature $\left(T H_{n}\right)(x, t)$ the Hermite temperature of order $n$ and denote it by $\mathscr{H}_{n}(x, t)$. In fact, we have

$$
\begin{aligned}
\mathscr{H}_{n}(x, t) & =\left\langle H_{n}(y), e^{-i x y-t|y|^{2}}\right\rangle \\
& =H_{n}(-D) E(x, t),
\end{aligned}
$$

so that $\mathscr{H}_{n}(x, t)\{E(x, t)\}^{-1}$ is a polynomial of degree $n$. Although the orthogonality of $\mathscr{H}_{n}(x, t)$ is not valid any longer they still satisfy the following: 


$$
\begin{aligned}
\left\langle\mathscr{H}_{n}(x, t), \mathscr{H}_{m}(x, t)\right\rangle: & =\int_{\boldsymbol{R}} \mathscr{H}_{n}(x, t) \mathscr{H}_{m}(x, t) d x \\
& =\int H_{n}(x) H_{m}(x) e^{-2 t|x|^{2}} d x \\
& = \begin{cases}2^{n} n ! \sqrt{\pi}, & \text { if } m=n, \\
0, & \text { if } m \neq n\end{cases}
\end{aligned}
$$

when $t=\frac{1}{2}$.

Now we will show that if a temperature satisfies some growth condition at inifinity, it can be expanded via Hermite temperatures as follows:

Theorem 5.2. Let $T>\frac{1}{2}$ and $u(x, t)$ be a temperature on $\mathbb{R} \times(0, T)$ satisfying that there exist $M>0, N>0$ and $C>0$ such that

$$
|u(x, t)| \leq C\langle x\rangle^{M_{t}-N}, \quad x \in \mathbb{R}, 0<t<T .
$$

Then it can be uniquely represented as

$$
u(x, t)=\sum_{n=0}^{\infty} a_{n} \mathscr{H}_{n}(x, t)
$$

Here, the coefficient $a_{n}$ is given by

$$
a_{n}=\frac{1}{2^{n} n ! \sqrt{\pi}} \int_{-\infty}^{\infty} u\left(x, \frac{1}{2}\right) \mathscr{H}_{n}\left(x, \frac{1}{2}\right) e^{-x^{2}} d x
$$

and satisfies that

$$
\left|a_{n}\right| \leq C(1+n)^{L}, \quad n \in N_{0},
$$

for some $L>0$ and $C>0$.

Proof. For a positive integer $m$ we put

$$
f(t)= \begin{cases}t^{m-1} /(m-1) !, & t \geq 0, \\ 0, & t<0 .\end{cases}
$$

Multiplying $f$ with a suitable $C^{\infty}$ function with compact support we obtain functions $v(t)$ and $w(t)$ with

$$
v(t)= \begin{cases}f(t), & t \leq T / 4, \\ 0, & t \geq T / 2,\end{cases}
$$


and supp $w \subset[T / 4, T / 2]$ such that

$$
\left(\frac{d}{d t}\right)^{m} v(t)=\delta(t)+w(t)
$$

where $\delta$ is the Dirac measure.

Now take the integer $m \geq N+2$ where $N$ is the constant in (5.4) and consider functions on $R \times(0, T / 2)$

$$
G(x, t)=\int_{0}^{T / 2} u(x, t+s) v(s) d s
$$

and

$$
H(x, t)=\int_{0}^{T / 2} u(s, t+s) w(s) d s
$$

Then they are all temperatures on $\boldsymbol{R} \times(0, T / 2)$ and satisfy

$$
|G(x, t)| \leq C\langle x\rangle^{M}, \quad x \in R, 0<t<\frac{T}{2}
$$

and

$$
|H(x, t)| \leq C\langle x\rangle^{M}, \quad x \in \boldsymbol{R}, 0<t<\frac{T}{2}
$$

Moreover, $G(x, t)$ and $H(x, t)$ can be continuously extended to $\boldsymbol{R} \times[0, T / 2)$ and

$$
\begin{aligned}
(-1)^{m} \frac{\partial^{2 m}}{\partial x^{2 m}} G(x, t) & =\left(-\frac{\partial}{\partial t}\right)^{m} G(x, t) \\
& =u(x, t)+H(x, t) .
\end{aligned}
$$

Define $g(x)=G(x, 0)$ and $h(x)=H(x, 0)$. Then $g$ and $h$ are continuous and

$$
|g(x)| \leq C\langle x\rangle^{M}, \quad|h(x)| \leq C\langle x\rangle^{M}, \quad x \in R .
$$

This implies that $g$ and $h$ can be regarded as tempered distributions. By the uniqueness theorem for temperatures (see [16]) we have

$$
\begin{aligned}
& G(x, t)=\int E(x-y, t) g(y) d y=(g * E)(x, t), \\
& H(x, t)=\int E(x-y, t) h(y) d y=(h * E)(x, t) .
\end{aligned}
$$


If we define a tempered distribution $u_{0}$ by

$$
u_{0}=(-1)^{m} \frac{\partial^{2 m}}{\partial x^{2 m}} g(x)-h(x)
$$

then

$$
\begin{aligned}
u_{0} * E & =\left[(-1)^{m} \frac{\partial^{2 m}}{\partial x^{2 m}} g-h\right] * E \\
& =(-1)^{m} \frac{\partial^{2 m}}{\partial x^{2 m}} G(x, t)-H(x, t) \\
& =u(x, t),
\end{aligned}
$$

which implies that the initial value $u\left(x, 0^{+}\right)$of $u(x, t)$ is given by the tempered distribution $u_{0}$. Since the inverse Fourier transform $\mathscr{F}^{-1}\left(u_{0}\right)$ of $u_{0}$ is still a tempered distribution we can write it as

$$
\mathscr{F}^{-1}\left(u_{0}\right)=\sum_{n=0}^{\infty} a_{n} H_{n}(x)
$$

for some sequence $a_{n}$ such that $\left|a_{n}\right| \leq C(1+n)^{L}, n \in N_{0}$. Now applying integral transformation we have

$$
T\left(\mathscr{F}^{-1}\left(u_{0}\right)\right)=u_{0} * E=u(x, t)
$$

and

$$
\begin{aligned}
T\left(\sum_{n=0}^{\infty} a_{n} H_{n}(x)\right) & =\sum_{n=0}^{\infty} a_{n}\left(T H_{n}\right)(x, t) \\
& =\sum_{n=0}^{\infty} a_{n} \mathscr{H}_{n}(x, t),
\end{aligned}
$$

which gives (5.5). Using the orthogonality (5.3) we also have (5.6). This completes the proof.

\section{Acknowledgment}

The author would like to thank the referee and Professor Dohan Kim for their valuable comments. This paper was supported by Korea Research Foundation in 1998. 


\section{References}

[1] Aronszajn, N., Traces of analytic solutions of heat equation, Astérisque, 2-3 (1973), 5-68.

[2] Chung, S.-Y., Positive definite temperature functions and a correspondence to positive temperature functions, Proc. Royal Soc. Edinburgh, 127A (1997), 947-962.

[ 3 ] Chung, J., Chung, S.-Y., and Kim, D., Positive definite hyperfunctions, Nagoya Math. J., 140 (1995), 139-149.

[4] Chung, S.-Y. and Kim, D., An example of nonuniqueness of the heat equation, Comm. Part. Diff. Equa., 19 (1994), 1257-1261.

[5] - Distributions with exponential growth and Bochner-Schwartz theorem for Fourier hyperfunction, Publ. RIMS, Kyoto Univ., 31 (1995), 829-845.

[6] Gelfand, I. M. and Shilov, G. E., Generalized functions II, Acad. Press, New York,1967.

[7] Hörmander, L., The analysis of linear partial differential operators I, SpringerVerlag, Berlin-New York, 1983.

[ 8 ] Haimo, D. T., Generalized temperature functions, Duke Math.J., 33 (1966), 305-322.

[9] - Widder temperature representations, J. Math. Analy. Appl., 41 (1973), 170-178.

[10] Kim, K. W., Chung, S.-Y., and Kim, D., Fourier hyperfunctions as the boundary values of smooth solutions of heat equations, Publ. RIMS, Kyoto Univ., 29 (1993), 289-300.

[11] Lebedev, N. N., Special functions and their applications, Dover Publications, 1972.

[12] Matsuzawa, T., A calculus approach to hyperfunctions II, Trans. Amer. Math. Soc., 313 (1990), 619-654.

[13] Strichartz, R., A guide to distribution theory and Fourier transforms, CRC Press, London, 1994.

[14] Stein, E. M. and Weiss, G., Introduction to Fourier analysis on Euclidean space, Princeton Univ., Princeton, 1975.

[15] Widder, D. V., Some analysis from classical analysis in the theory of heat equation, Arch. Rational. Mech. Anal., 21 (1966), 108-119.

[16] - Heat equation, Acad. Press, New York, 1975. 
